The Astrophysical Journal, 637:322-332, 2006 January 20

(C) 2006. The American Astronomical Society. All rights reserved. Printed in U.S.A.

\title{
THE EFFECT OF COHERENT STRUCTURES ON STOCHASTIC ACCELERATION IN MHD TURBULENCE
}

\author{
KASPAR ARZNER \\ Paul Scherrer Institut, Villigen, CH-5232 Villigen PSI, Switzerland; arzner@astro.phys.ethz.ch \\ Bernard Knaepen, Daniele Carati, and Nicolas Denewet \\ Université libre de Bruxelles, Brussels, Belgium; bknaepen@ulb.ac.be \\ AND \\ LOUKAS VLAHOS \\ Department of Physics, Aristotle University of Thessaloniki, Thessaloniki 54124, Greece; vlahos@astro.auth.gr \\ Received 2005 August 18; accepted 2005 September 22
}

\begin{abstract}
We investigate the influence of coherent structures on particle acceleration in the strongly turbulent solar corona. By randomizing the Fourier phases of a pseudospectral simulation of isotropic magnetohydrodynamic (MHD) turbulence $(\operatorname{Re} \sim 300)$ and tracing collisionless test protons in both the exact-MHD and phase-randomized fields, it is found that the phase correlations enhance the acceleration efficiency during the first adiabatic stage of the acceleration process. The underlying physical mechanism is identified as the dynamical MHD alignment of the magnetic field with the electric current, which favors parallel (resistive) electric fields responsible for initial injection. Conversely, the alignment of the magnetic field with the bulk velocity weakens the acceleration by convective electric fields $-\boldsymbol{u} \times \boldsymbol{b}$ at a nonadiabatic stage of the acceleration process. We point out that nonphysical parallel electric fields in random-phase turbulence proxies lead to artificial acceleration and that the dynamical MHD alignment can be taken into account on the level of the joint two-point function of the magnetic and electric fields and is therefore amenable to Fokker-Planck descriptions of stochastic acceleration.
\end{abstract}

Subject headings: acceleration of particles — methods: numerical — MHD

\section{INTRODUCTION}

Turbulent electromagnetic fields can act as particle accelerators in astrophysical situations such as solar flares (Miller et al. 1997; Benz 2003). The turbulence is governed by nonlinear fluid equations, and the turbulent fields therefore deviate from a simple Gaussian field (Adler 1981), in which the Fourier phases are at random, and the field is completely specified by its two-point function. In real space, the turbulent phase correlations manifest in coherent structures, with alignment of magnetic and velocity fields (Pouquet et al. 1986), and in non-Gaussian higher order correlations (McComb 1990; Falkovich et al. 2001).

The latter are not captured by traditional stochastic acceleration theory, which invokes the two-point functions of the magnetic field to predict the particle diffusion coefficients (e.g., Urch 1977; Achterberg 1981; Karimabadi et al. 1990; Urch 1991; Bykov \& Toptygin 1993; Schlickeiser \& Miller 1998; Schlickeiser 2002). On the numerical side, some studies (e.g., Mace et al. 2000; Paesold et al. 2003; Arzner \& Vlahos 2004; Arzner et al. 2005) have worked with random-phase turbulence proxies and therefore neglected coherent structures, while others (e.g., Matthaeus et al. 1984; Dmitruk et al. 2003) used simulated MHD fields and took them into account.

The presence of coherent structures may have conflicting effects on the acceleration efficiency (Dmitruk et al. 2003). On the one hand, the coherent structures may act as traps and detain particles on their way to high energies. On the other, coherent structures may host large electric fields, which help acceleration. Which of the two effects prevails is hard to predict on theoretical grounds and depends on the topology and size of the coherent structures. A numerical exploration is thus needed to capture the whole diversity of possible orbit behavior. The present article reports on relativistic test particle simulations in turbulent MHD electromagnetic fields, following a similar approach as Dmitruk et al. (2003). By applying an artificial phase randomization to the electromagnetic fields we then study the effect of coherent structures on test particle acceleration and compare it with the Gaussian field limit.

The astrophysical realization envisaged by the present simulation is proton acceleration in solar flares, with emphasis on the high-energy regime in which adiabaticity of the orbit breaks down. Scaling to high-energy electrons or heavier ions is in principle possible but not explicitly discussed. The paper is organized as follows. Section 2 describes the MHD fields and their randomization, and $\S 3$ describes the numerical generation of particle orbits and the physical approximations made in doing so. Section 4 presents the particle results, followed by a discussion of the acceleration mechanism $(\S 5)$. Section 6 contains a summary of the simulation results and a discussion of their validity and implications.

\section{ELECTROMAGNETIC FIELDS}

\subsection{Random versus Coherent Fields}

As indicated in the introduction, the focus of this paper is on the role of turbulent coherent structures on particle acceleration. Here we assume that particles are only subject to an electric field $\boldsymbol{e}$ and a magnetic field $\boldsymbol{b}$ through the (relativistic) Lorentz force (we will refer to a couple $\boldsymbol{e}$ and $\boldsymbol{b}$ as an "accelerator"). The electric field is taken according to MHD,

$$
\boldsymbol{e}=-\boldsymbol{u} \times \boldsymbol{b}+\eta \boldsymbol{j}, \quad \nabla \times \boldsymbol{b}=\boldsymbol{j},
$$

where $\boldsymbol{u}$ is the bulk velocity, $\boldsymbol{j}$ is the electric current, and $\eta$ is the magnetic diffusivity. In equation (1) and from now on we use Alfvén units, i.e., the magnetic field is scaled to the Alfvén velocity, $\boldsymbol{b}=\boldsymbol{B} /\left(\mu_{0} \rho\right)^{1 / 2}$, and measured in the same units as the 
TABLE 1

Turbulence Characteristics of the Velocity and Magnetic Fields Used To Construct $(\mathcal{E B})_{\text {Dns }}$

\begin{tabular}{|c|c|}
\hline Parameter & Value \\
\hline Resolution ............................... & $256^{3}$ \\
\hline Box size $\left(l_{x} \times l_{y} \times l_{z}\right) \ldots \ldots \ldots \ldots$ & $2 \pi \times 2 \pi \times 2 \pi$ \\
\hline rms velocity $u=\left(\left\langle u_{i} u_{i}\right\rangle / 3\right)^{1 / 2} \ldots \ldots \ldots \ldots$ & 2.20 \\
\hline rms magnetic field $b=\left(\left\langle b_{i} b_{i}\right\rangle / 3\right)^{1 / 2}$. & 2.39 \\
\hline 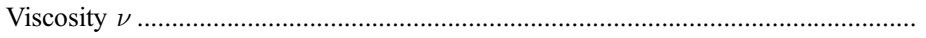 & 0.006 \\
\hline 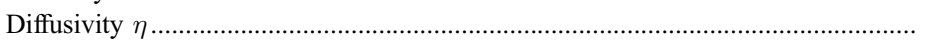 & 0.006 \\
\hline Kinetic dissipation $\epsilon_{u} \ldots \ldots \ldots \ldots \ldots \ldots . . . .$. & 15.28 \\
\hline Magnetic dissipation $\epsilon_{b}$ & 20.57 \\
\hline 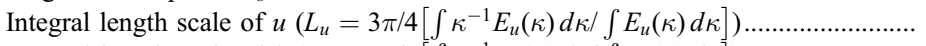 & 0.79 \\
\hline 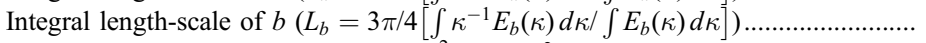 & 0.71 \\
\hline 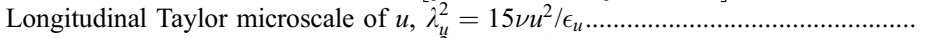 & 0.169 \\
\hline 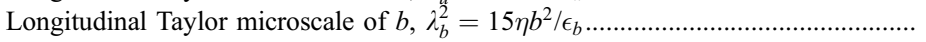 & 0.158 \\
\hline 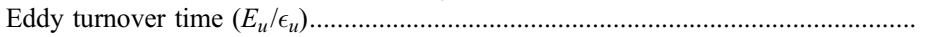 & 0.421 \\
\hline
\end{tabular}

Notes. $-E_{u}(\kappa)$ and $E_{b}(\kappa)$ designate the velocity and magnetic field spectra, e.g., $E_{u}=\frac{3}{2} u^{2}=\int E_{u}(\kappa) d \kappa$. Angle brackets denote a spatial average.

bulk velocity. Another assumption of the present study is that the particles' motion takes place in a cubic domain with periodic boundary conditions. This implies that at any instant the MHD fields can be viewed as superpositions of waves and decomposed in Fourier modes:

$$
\begin{aligned}
& \boldsymbol{u}(\boldsymbol{x})=\sum \tilde{\boldsymbol{u}}(\boldsymbol{k}) e^{i \boldsymbol{k} \cdot \boldsymbol{x}}, \\
& \boldsymbol{b}(\boldsymbol{x})=\sum \tilde{\boldsymbol{b}}(\boldsymbol{k}) e^{i \boldsymbol{k} \cdot \boldsymbol{x}} .
\end{aligned}
$$

In the above expansions, $\tilde{\boldsymbol{u}}(\boldsymbol{k})$ and $\tilde{\boldsymbol{b}}(\boldsymbol{k})$ are complex numbers that satisfy $\tilde{\boldsymbol{u}}(-\boldsymbol{k})=\tilde{\boldsymbol{u}}^{*}(\boldsymbol{k}), \tilde{\boldsymbol{b}}(-\boldsymbol{k})=\tilde{\boldsymbol{b}}^{*}(\boldsymbol{k})$ and can be written as

$$
\tilde{\boldsymbol{u}}(\boldsymbol{k})=\tilde{\boldsymbol{u}}_{r}(\boldsymbol{k}) e^{i \alpha(\boldsymbol{k})}, \quad \tilde{\boldsymbol{b}}(\boldsymbol{k})=\tilde{\boldsymbol{b}}_{r}(\boldsymbol{k}) e^{i \beta(\boldsymbol{k})},
$$

with real-valued $\boldsymbol{u}_{r}, \boldsymbol{b}_{r}, \alpha$, and $\beta$. Constructing a numerical field like equation (4) thus requires information both on the modulus and the phases of its Fourier modes. In homogeneous and isotropic turbulence, the modulus of Fourier modes is usually set to given values that depend only on the norm of the corresponding wave vector to mimic a given energy spectra. Assigning appropriate phases to the Fourier modes is a much more complex task. In a real turbulent field, these phases have to be correlated in such a way as to produce a field with subtle and intricate structures. Prescribing them numerically represents the same complexity as prescribing the electromagnetic field at each point in space and is thus not practical. The most simple way of circumventing this problem is to assign random numbers in the interval $(-\pi, \pi)$ to the phases and impose a given distribution, most often the uniform distribution to respect isotropy. The fields generated can then be qualified as stochastic but still have a prescribed energy spectra. More realistic phases can be obtained by generating the electromagnetic field through direct numerical simulations (DNSs) of the MHD equations,

$$
\begin{aligned}
\partial_{t} u_{i} & =-\partial_{i}(p / \rho)-u_{j} \partial_{j} u_{i}+b_{j} \partial_{j} b_{i}+\nu \Delta u_{i}, \\
\partial_{t} b_{i} & =-u_{j} \partial_{j} b_{i}+b_{j} \partial_{j} u_{i}+\eta \Delta b_{i},
\end{aligned}
$$

where $p$ is the sum of the kinematic and magnetic pressures and $\nu$ is the kinematic viscosity. The magnetic field contains no mean contribution. Since we assume incompressibility, $p$ is obtained by solving a Poisson equation and is not an independent variable (e.g., McComb 1990). Although the initial condition used in equa- tions (5) and (6) consists of fields with random phases, it is observed that after some time, the resulting electromagnetic field exhibits coherency that deviates strongly from its random phases counterpart (e.g., Biskamp \& Müller 1999). Although the DNS approach is very appealing, it should be stressed that it is very costly and that the values of Reynolds numbers observed in typical turbulent systems are out of reach of present-day supercomputers. In that sense, the fields obtained through DNS also constitute models for real-life turbulence.

Our objective is here to study two kinds of accelerators: a random phases one and a coherent one obtained through DNS. To make things consistent, we construct both accelerators in such a way that they have exactly the same spectra and only differ through the value of the phases of their Fourier modes. As a shorthand, the random phases accelerator will be denoted $(\mathcal{E B})_{\text {STO }}$ while the DNS accelerator will be denoted $(\mathcal{E B})_{\mathrm{DNS}}$. In the next section we detail the construction of $(\mathcal{E B})_{\mathrm{STO}}$ and $(\mathcal{E} \mathcal{B})_{\mathrm{DNS}}$.

\subsection{MHD Simulations}

In order to obtain a suitable DNS electromagnetic field, we solve equations (5) and (6) using a pseudo-spectral code in a cubic domain of side length $l_{x}=l_{y}=l_{z}=2 \pi$ and impose periodic boundary conditions. Our simulation code is dealiased and uses a fourth-order accurate Runge-Kutta time advancement scheme. As explained above, the initial condition for $u_{i}$ and $b_{i}$ are random phases fields of the form (4), whose spectra match the one observed in the Comte-Bellot-Corrsin grid turbulence experiment at stage 2 (Comte-Bellot \& Corrsin 1971) (this choice of spectra is motivated by the fact that the corresponding turbulence can be resolved with a resolution of $256^{3}$ Fourier modes). The only extra requirement imposed on the Fourier modes is incompressibility, $\boldsymbol{k} \cdot \tilde{\boldsymbol{b}}(\boldsymbol{k})=\boldsymbol{k} \cdot \tilde{\boldsymbol{u}}(\boldsymbol{k})=0$. As time advances, the flow becomes "physical" and we stop the simulation when the skewness of the velocity derivative $\partial u_{1} / \partial x_{1}$ reaches a quasi constant value (Batchelor 1982; McComb 1990; Gotoh et al. 2002; Verma 2004). This criteria is standard and indicates that turbulence has had time to develop. Here, the initialization phase has taken about $2.5 t_{\mathrm{A}}$ where $t_{\mathrm{A}}$ is the Alfvén time defined by $t_{\mathrm{A}}=L / v_{\mathrm{A}}$, with $L$ the (magnetic or velocity) integral scale-length, and $v_{\mathrm{A}}^{2}=\left\langle|\boldsymbol{b}|^{2}\right\rangle / 3$ (from here on, angle brackets denote a spatial average). Note that $v_{\mathrm{A}}$ is determined by the (rms) magnetic fluctuations and not by a background magnetic field. Some of the characteristics of the flow at $2.5 t_{\mathrm{A}}$ are listed in Table 1 . There is approximate equipartition 

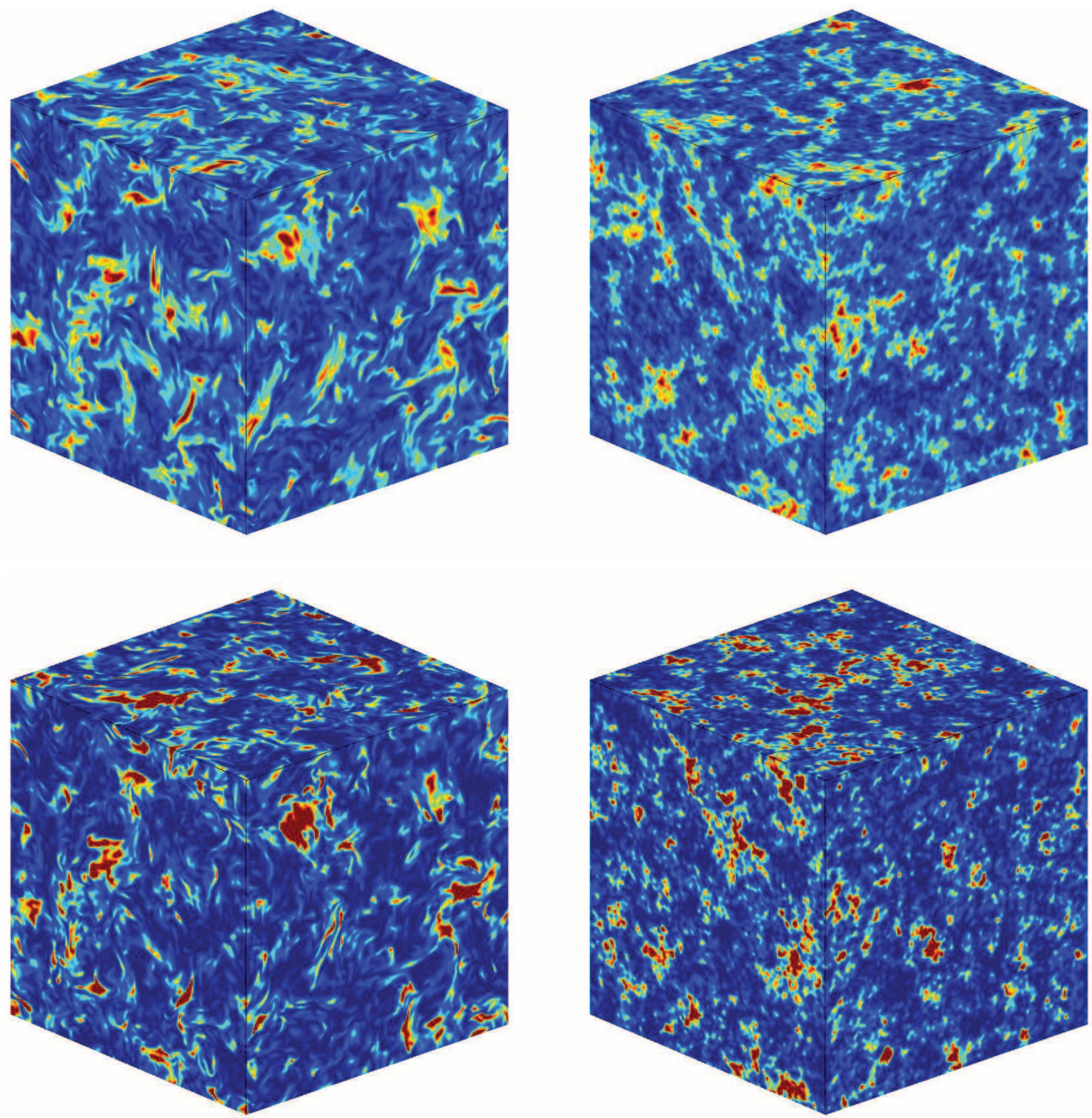

FIG. 1.-Magnetic (top) and electric (bottom) energy densities of the $(\mathcal{E B})_{\text {DNS }}($ left $)$ and $(\mathcal{E B})_{\text {Sto }}$ (right) fields, similar as in Dmitruk et al. (2003). Blue and red regions indicate, respectively, low and high values.

between $|\boldsymbol{b}|^{2}$ and $|\boldsymbol{u}|^{2}$, and both dissipate at the same rate (magnetic Prandtl number $\sim 1$ ), so that the magnetic and velocity characteristic scales are similar. The Reynolds number and magnetic Reynolds number are about 300. The magnetic field extracted from the DNS at this final time and the electric field obtained through equation $(1)$ constitute the accelerator $(\mathcal{E B})_{\text {DNS. }}$.

To construct $(\mathcal{E B})_{\mathrm{STO}}$ we modify the phases of $\tilde{\boldsymbol{b}}(\boldsymbol{k})$ and $\tilde{\boldsymbol{u}}(\boldsymbol{k})$ according to

$$
\alpha_{i}(\boldsymbol{k}) \rightarrow \alpha_{i}(\boldsymbol{k})+\phi(\boldsymbol{k}), \quad \beta_{i}(\boldsymbol{k}) \rightarrow \beta_{i}(\boldsymbol{k})+\varphi(\boldsymbol{k}),
$$

where $\phi(\boldsymbol{k})$ and $\varphi(\boldsymbol{k})$ are random numbers distributed uniformly in the interval $(-\pi, \pi)$ and which satisfy $\phi(\boldsymbol{k})=-\phi(-\boldsymbol{k})$ and $\varphi(\boldsymbol{k})=-\varphi(-\boldsymbol{k})$. The electric field is then computed from equation (1). The above transformation ensures that (1) the phases of the new fields $(\mathcal{E B})_{\mathrm{STO}}$ contain a highly random part; (2) the spectra of $\boldsymbol{u}$ and $\boldsymbol{b}$ remain unchanged; (3) the divergence of $\boldsymbol{b}$ remains equal to zero; (4) the ideal MHD condition $\boldsymbol{e} \cdot \boldsymbol{b}=0$ is preserved if $\eta=0$, so that the randomization does not introduce parallel electric fields.

\subsection{Intrinsic Properties of the Accelerators $(\mathcal{E B})_{\mathrm{DNS}}$ and $(\mathcal{E B})_{\mathrm{STO}}$}

The whole purpose of building an accelerator using DNS of the MHD equations is to obtain an electromagnetic configuration that has more characteristic features of turbulence than only the correct energy spectra. In this section we describe some of the differences between $(\mathcal{E B})_{\mathrm{DNS}}$ and $(\mathcal{E B})_{\mathrm{STO}}$ that are relevant from the point of view of turbulence and particle acceleration. See Verma (2004) for a recent review on MHD turbulence.

Figure 1 presents three-dimensional views of the magnetic $\left(|\boldsymbol{b}|^{2}\right)$ and electric $\left(|\boldsymbol{e}|^{2}\right)$ energy densities. Although this corresponds only to a visual impression, it appears clear from the graphs that the electromagnetic field composing $(\mathcal{E B})_{\mathrm{DNS}}$ hosts more coherent, filamentary structures than the one composing $(\mathcal{E B})_{\mathrm{STO}}$. In the latter, structures appear noisier and span regions of a smaller extent.

Also, it appears from Figure 1 that the electric field has more extreme values (dark red) than the magnetic field, as already noticed by Dmitruk et al. (2003). More quantitative information on this can be obtained from the distributions (one-point functions) 

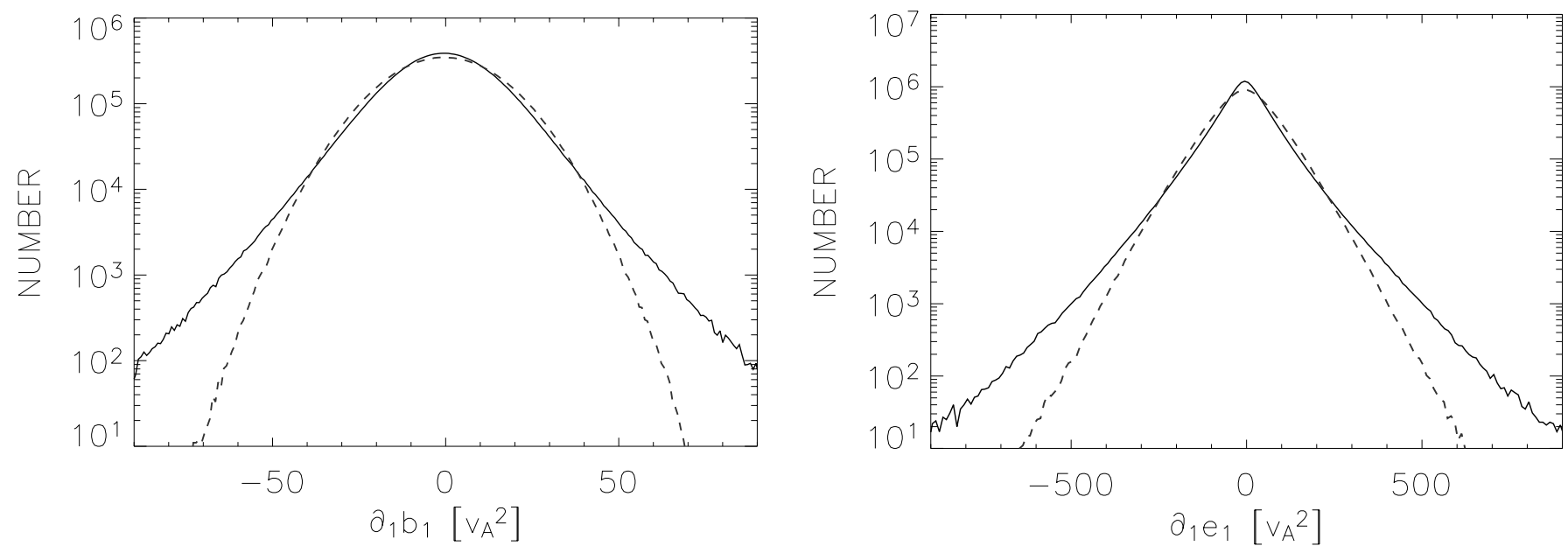

FIG. 2.- Histograms for the longitudinal derivatives of magnetic (left) and electric (right) field components: DNS (solid line); random phase (dashed line).

of individual field components. In the case of $(\mathcal{E B})_{\mathrm{STO}}$ it is observed, as expected, that $u_{1}$ and $b_{1}$ have a nearly Gaussian distribution (other components behave similarly). The distribution of $e_{1}$ [again for $(\mathcal{E B})_{\mathrm{STO}}$ ] is very close to a zeroth order modified Bessel function of the second kind, which decays exponentially for large values of its argument. This is again expected, since at high Reynolds number $e_{1}$ is dominated by the term $-\boldsymbol{u} \times \boldsymbol{b}$ which behaves as the product of two Gaussian variants for $(\mathcal{E B})_{\mathrm{STO}}$. In the case of $(\mathcal{E B})_{\mathrm{DNS}}$, the distribution of the field components are similar to their random-phase equivalents. Only for large values do they exhibit moderately pronounced tails resulting from higher intermittency.

A better measure of coherency is contained in the distributions of the longitudinal derivatives such as $\partial_{1} b_{1}$ and $\partial_{1} e_{1}$ (see Gotoh et al. 2002) (Fig. 2). Again, the second and third components behave similarly and are therefore not shown. In the DNS case, both $\partial_{1} b_{1}$ and $\partial_{1} e_{1}$ depart significantly from their random-phase equivalents. The distributions exhibit a large number of extreme values (tails in the distribution). These events allow coherent structures to be present in the signal since they correspond to regions of space where sharp transitions "shaping" the signal can occur. The strong differences in this diagnostic between the DNS and random phases case reflect the marked visual differences observed in Figure 1.

The coherent structures also manifest in the longitudinal structure functions (Kolmogoroff 1941a, 1941b; McComb 1990; Frisch 1995; Sorriso-Valvo et al. 2000; Falkovich et al. 2001; Gotoh et al. 2002; Cho et al. 2002) which are a natural extension of the longitudinal derivatives discussed above. The magnetic longitudinal structure functions are defined by $D_{p}(\boldsymbol{r})=\langle|[\boldsymbol{b}(\boldsymbol{r}+\boldsymbol{x})-$ $\left.\boldsymbol{b}(\boldsymbol{x})]\left.\cdot \hat{\boldsymbol{r}}\right|^{p}\right\rangle$ and measure the variation of the $p$-th moment of the longitudinal magnetic field increment with distance $r$ (hat denotes a unit vector). Figure 3 (inset) shows the distribution of these increments along $\hat{r}_{i}$, averaged over $i=1,2$, and 3 . With increasing $r$, the increments become decorrelated and their distribution becomes Gaussian. The transition can be monitored by means of the kurtosis $\kappa(r)=D_{4}(r) / D_{2}(r)^{2}$; Figure 3 (diamonds) shows the DNS-to-STO kurtosis ratio as a function of $r$. There is a clear systematic excess of the DNS kurtosis at small $r$, and one may infer from Figure 3 that on scales $r>1$, the DNS and STO fields behave similarly. In particular, the DNS field becomes independent at separations that are long compared to the integral length-scale of $\boldsymbol{u}$ and $\boldsymbol{b}$ (Table 1).

Another distinction between $(\mathcal{E B})_{\mathrm{DNS}}$ and $(\mathcal{E B})_{\mathrm{STO}}$, which will prove helpful in understanding the particle acceleration, is the relative orientation of $\boldsymbol{b}$ with respect to $\boldsymbol{u}$ and $\boldsymbol{j}$. The nonlinear MHD equations (6) and (5) tend to align $\boldsymbol{b}$ with $\boldsymbol{j}$ in order to minimize the $\boldsymbol{j} \times \boldsymbol{b}$ force. The (approximate, $R e_{b}=283$ ) frozen-in condition tends, then, to align $\boldsymbol{b}$ with $\boldsymbol{u}$ (Biskamp 1993; Dar 1998; Debliquy et al. 2005). This preference for alignment is demonstrated in Figure 4, showing the distribution of the orientation cosines $\hat{\boldsymbol{b}} \cdot \hat{\boldsymbol{u}}$ and $\hat{\boldsymbol{b}} \cdot \hat{\boldsymbol{j}}$ within the simulation cube. The DNS distributions (black line) accumulate at the extreme values \pm 1 , corresponding to $\pm 180^{\circ}$, more pronounced for $\hat{\boldsymbol{b}} \cdot \hat{\boldsymbol{j}}$ than for $\hat{\boldsymbol{b}} \cdot \hat{\boldsymbol{u}}$. After the phase randomization is applied (gray line), this accumulation vanishes and the distribution of $\hat{\boldsymbol{b}} \cdot \hat{\boldsymbol{j}}$ (bottom) become isotropic. The residual anticorrelation between $\hat{\boldsymbol{b}}$ and $\hat{\boldsymbol{u}}$ (top, gray line), corresponding to a negative cross-helicity, is dominated by few

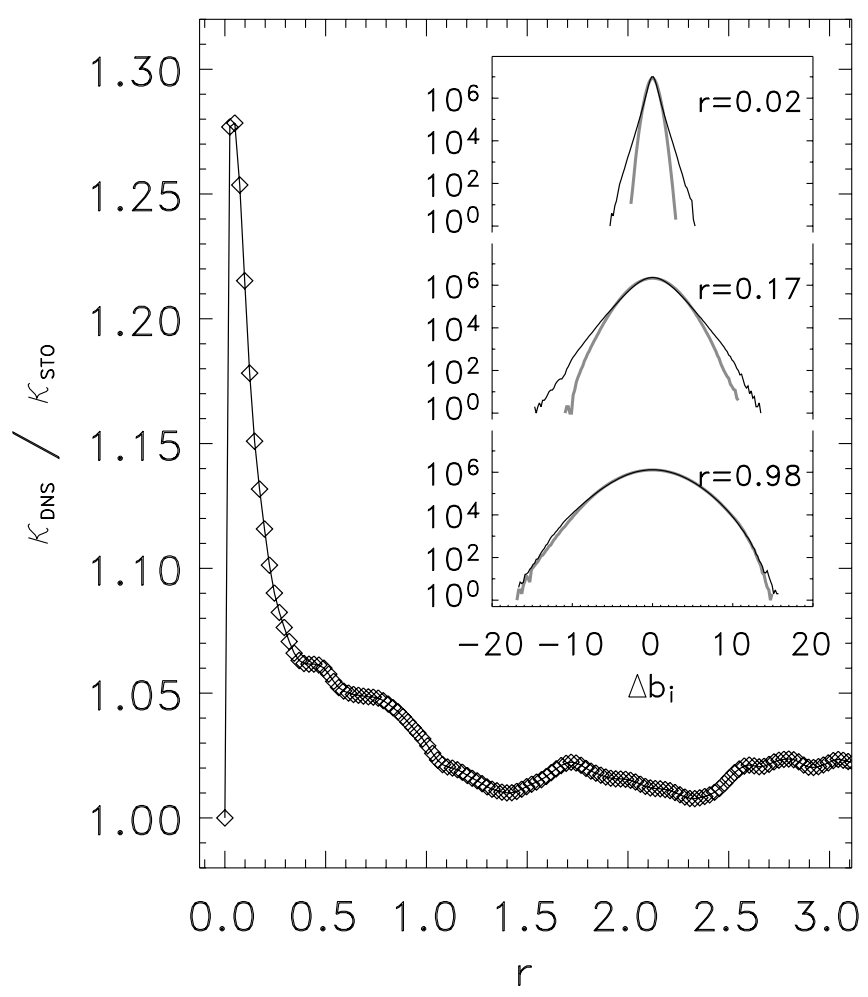

FIG. 3.-Excess of the kurtosis of $\Delta b_{i}=b_{i}\left(\boldsymbol{x}+r \hat{e}_{i}\right)-b_{i}(\boldsymbol{x})$ between DNS and STO fields, averaged over $i=1,2,3$ (diamonds). Inset: Some representative histograms of $\Delta b_{i}$ (average over $i=1,2,3$ ); the black and gray lines represent DNS and STO cases, respectively. See text. 

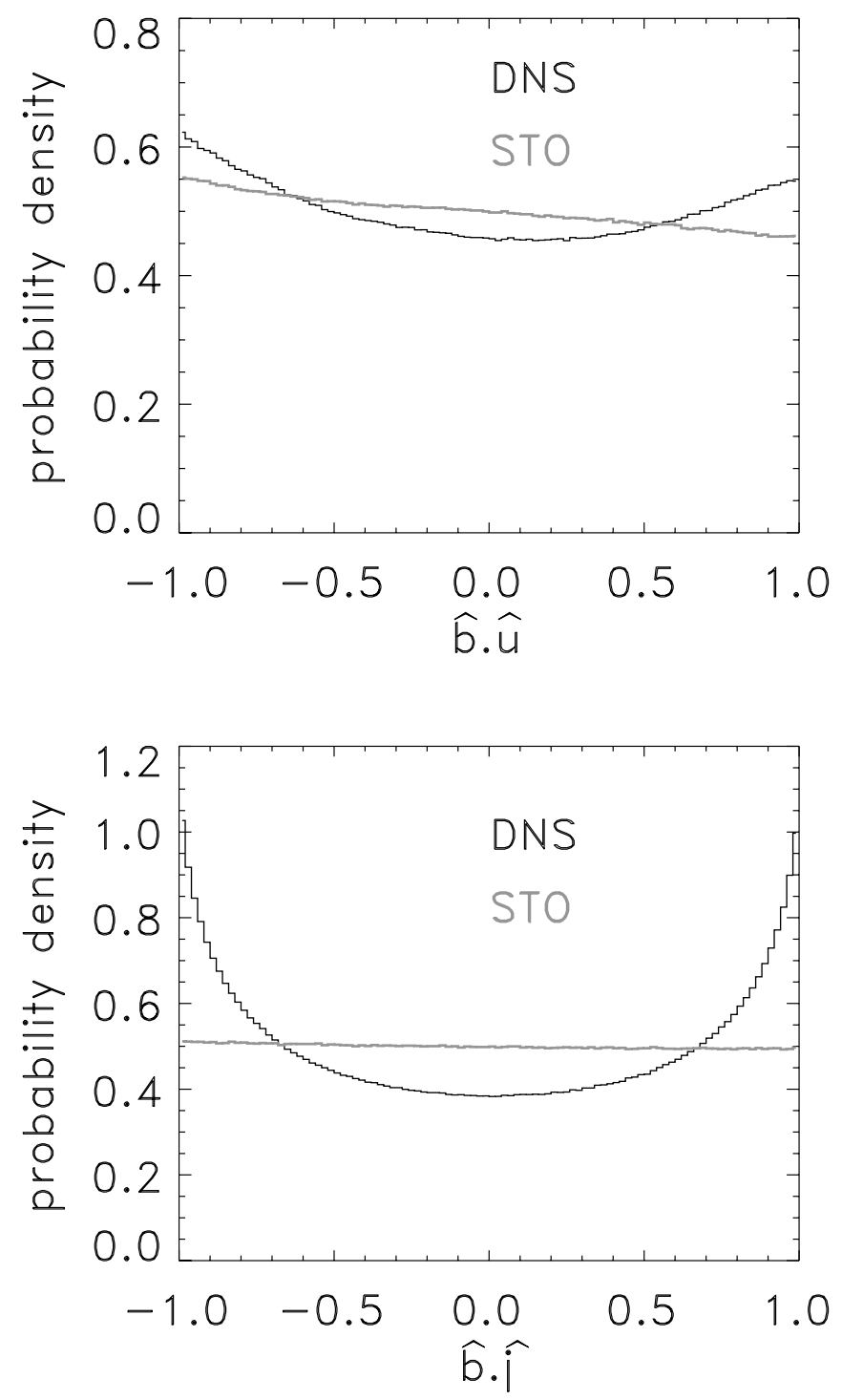

FIG. 4.-Distributions of the angle cosines between $\boldsymbol{b}$ and $\boldsymbol{u}$ (top), and $\boldsymbol{b}$ and $\boldsymbol{j}$ (bottom).

small- $|\boldsymbol{k}|$ modes, and is within statistical errors. Note that for the mixed-field correlations, the coherent structures already manifest at the level of $\boldsymbol{b} \cdot \boldsymbol{u}$ and $\boldsymbol{b} \cdot \boldsymbol{j}$, whereas one has to go to higher order moments if $\boldsymbol{b}$ and $\boldsymbol{u}$ are separately considered (Fig. 3).

\section{PARTICLE ORBITS}

\subsection{Physical Environment}

An ensemble of collisionless charged particles is amenable to the MHD description if their orbits can be described by the drift velocity $\boldsymbol{e} \times \boldsymbol{b} /|\boldsymbol{b}|^{2}+\boldsymbol{v}_{\|}$, where the double bars refer to the local magnetic field. The drift velocity then equals the MHD bulk velocity $\boldsymbol{u}$. This description requires that the Larmor radius be much smaller than the radius of curvature of the magnetic field and that $v_{\|}$be smaller than the Alfvén velocity (e.g., Littlejohn 1982; Büchner \& Zelenyi 1989). In the quiet solar corona, the ordering $v<v_{\text {A }}$ holds true for both electrons and ions. During solar flares, when turbulence arises, a small fraction of the particles reach relativistic energies, and it is this high-energy population that is treated here as test particles. The nonenergetic bulk provides the MHD acceleration environment. The acceleration to relativistic energies is known to be rather rapid. In impulsive flares, hard $\mathrm{X}$-ray signatures of relativistic electrons appear within fractions of a second, and gamma-ray signatures of relativistic protons - if any-appear within a few seconds (Miller et al. 1997). The MHD structures, in contrast, evolve on a much longer timescale.

The solar corona, in which acceleration is thought to occur, is a relatively collisionless environment. Assuming a magnetic field strength of $10^{-2} \mathrm{~T}$, the electron and proton gyro times are in the order of nanoseconds and microseconds, respectively. In contrast, the ion-electron collision time is in the order of tens of milliseconds, if we adopt a typical temperature $T_{e}=T_{i}=10^{6} \mathrm{~K}$ and density $10^{16} \mathrm{~m}^{-3}$. The particles of the background plasma thus perform many gyrations before a Coulomb collision is encountered, and this becomes even more true for high-energy test particles, because the collision rate rapidly decreases with energy (Dreicer 1959; Helander \& Sigmar 2002). While the background plasma relaxes toward a Maxwellian on timescales of tens of milliseconds, a relativistic test proton remains virtually collisionless.

For particles that move much faster than the background plasma, the MHD fields may be regarded as static. This requires $v \gg v_{\mathrm{A}}$. In the solar corona, $v_{\mathrm{A}}$ is in the order of $1000 \mathrm{~km} \mathrm{~s}^{-1}$, and the condition $v \gg v_{\mathrm{A}}$ is fulfilled for relativistic particles. However, acceleration begins at Alfvénic velocities, and the question arises whether a static approximation applies from the very beginning. This depends on the time spent at low velocities. As outlined above, this time is observationally known to be short compared to the MHD timescale in the real solar corona, and in our orbit simulations we will find that particles reach relativistic velocities within some $0.01 t_{\mathrm{A}}$. The accelerated particles therefore spend most of the simulation in a state with $v \gg v_{\mathrm{A}}$, and we set

$$
\boldsymbol{b}(\boldsymbol{x}, t)=\boldsymbol{b}\left(\boldsymbol{x}, t_{0}\right), \quad \boldsymbol{e}(\boldsymbol{x}, t)=\boldsymbol{e}\left(\boldsymbol{x}, t_{0}\right), \quad \forall t
$$

throughout the whole orbit simulation. The stationarity assumption (eq. [8]) admits conservation laws, which facilitate the numerical benchmarking and the physical understanding of the acceleration process. In this sense, equation (8) also represents a simplifying working hypothesis.

The test particles orbits are measured in the same units as the MHD fields. The dimensionless equations of motion read

$$
\begin{aligned}
\frac{d \boldsymbol{x}}{d t} & =\boldsymbol{v}, \\
\frac{d \gamma \boldsymbol{v}}{d t} & =\alpha(\boldsymbol{e}+\boldsymbol{v} \times \boldsymbol{b}),
\end{aligned}
$$

where $\gamma=\left(1-v^{2} / c^{2}\right)^{-1 / 2}$ is the Lorentz factor, and the effective charge $\alpha=\Omega t_{\mathrm{A}}$ defines the particle timescales relative to the MHD timescale (Dmitruk et al. 2003), with $\Omega$ the proper rms gyro frequency. Assuming a coronal magnetic field of $10^{-2}$ $\mathrm{T}$ and a density of $10^{16} \mathrm{~m}^{-3}$, we have $v_{\mathrm{A}}=2182 \mathrm{~km} \mathrm{~s}^{-1}=$ $0.0073 c$ (thus $c=137.61$ in numerical units), and focusing on protons as test particles, we set $\alpha=10^{3}$. For display purposes, time is measured in units of the (dimensionless) proper gyro time $t_{C}=2 \pi /\left(\alpha \sqrt{\left\langle|\boldsymbol{b}|^{2}\right\rangle}=3.6 /\left(\alpha v_{\mathrm{A}}\right)\right.$, corresponding to about $5 \times 10^{-6} \mathrm{~s}$ in real time. All test particles start out at random position and in random direction with initial velocity $v_{0}=0.2 v_{\mathrm{A}}$, representative of protons with $T=10^{7} \mathrm{~K}$. This ensures that the test protons can be considered as approximately collisionless, and that the initial Larmor radius is smaller than the numerical cell. The orbits are exactly integrated, without recourse to 

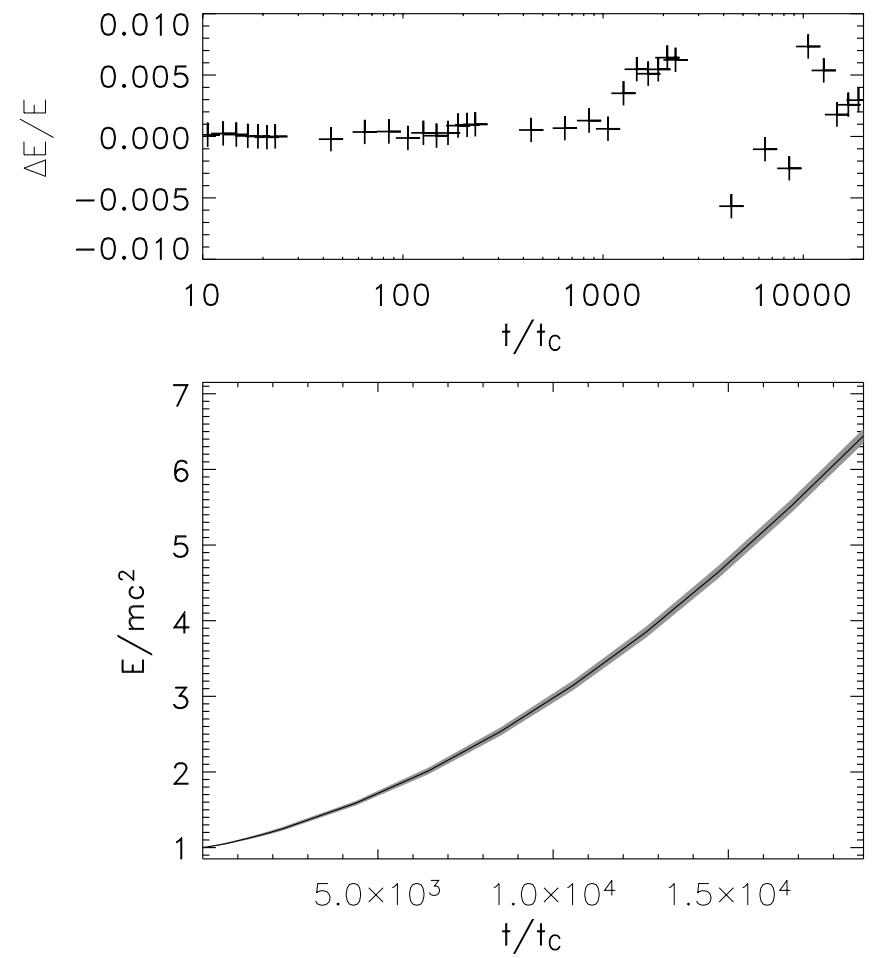

FIG. 5.-Benchmarking of energy conservation (eq. [11]). Top: Relative error along an example orbit. Bottom: Ensemble average, with the shaded region representing the $\pm 5 \sigma$ deviations. Time is measured in units of the proper rms gyro time $t_{C}$.

gyrokinetic approximations. This approach benefits from simplicity and rigor but allows only relatively small populations to be simulated over relatively short times. See $\S 6$ for further discussion of the limitations of the method.

\subsection{Numerical Implementation and Benchmarking}

Equations (9) and (10) are integrated by a fourth-order RungeKutta scheme with constant time step. The electromagnetic fields are taken from a snapshot of the pseudospectral MHD simulation, transformed to real space, and linearly interpolated to the actual particle positions. A parallel (distributed memory) implementation is used to enhance the computational power. The diagnostics includes energy histograms and the second moments of momentum and space displacements.

The static assumption (eq. [8]) implies that the vector potential evolves linearly with time and that the scalar potential $\phi$ is independent of time. Therefore, the identity

$$
\gamma c^{2}+\alpha \phi=H_{0}-\alpha \int d t \boldsymbol{v} \cdot \boldsymbol{e}_{\mathrm{sol}}
$$

must hold, where $\boldsymbol{e}_{\text {sol }}$ is the solenoidal $\left(\nabla \cdot \boldsymbol{e}_{\text {sol }}=0\right)$ part of the electric field and $H_{0}$ is the initial energy. Numerically, $\phi$ and $\boldsymbol{e}_{\text {sol }}$ are computed from the Fourier amplitudes $\tilde{\boldsymbol{e}}(\boldsymbol{k})$ by decomposition into parallel and perpendicular components with respect to $\boldsymbol{k}$. To make the decomposition unique, the potential is gauged such that $\langle\phi\rangle=0$. Equation (11) can be used to assess the numerical accuracy and to choose an appropriate time step. To this end, the right-hand side of equation (11) is numerically integrated along the trajectories and compared to the left-hand side (Fig. 5). The observed discrepancy $\Delta E$ depends on the time step $\Delta t$ and on the smoothness of the MHD fields; both small $\Delta t$ and smooth fields help to fulfill equation (11). In view of the fact that real acceleration increases the particles' kinetic energy by orders of magnitudes, an rms discrepancy of $1 \%$ on the $2 \sigma$ level between the left- and right-hand sides of equation (11) at the end of the simulation is considered as acceptable. This is achieved if $\Delta t<0.01 \times\left(\alpha v_{\mathrm{A}}\right)^{-1}$.

The Runge-Kutta integration scheme was also tested against a simpler leapfrog scheme, and it was shown that it provided a significant increase in precision.

\section{RESULTS}

In this section we summarize the outcome of the particle simulations, focusing on the second moments and correlation functions of the dynamical quantities $(\boldsymbol{x}, \boldsymbol{p})$. The average over particles is denoted by double angle brackets in order to distinguish it from the spatial average, denoted by single angle brackets. The particle ensembles comprise 50,000 particles.

\subsection{Real-Space Diffusion}

A first qualitative difference between the $(\mathcal{E B})_{\text {DNS }}$ and $(\mathcal{E B})_{\text {STO }}$ cases concerns the intermittency of their real-space trajectories. Figure 6 shows sample orbits in $(\mathcal{E B})_{\mathrm{DNS}}($ left $)$ and $(\mathcal{E B})_{\mathrm{STO}}$ (right) fields for times up to $t / t_{C} \leq 1.5 \times 10^{3}$. As can be seen, the $(\mathcal{E B})_{\text {DNS }}$ orbits tend to have long sojourns between clusters where particles are temporarily trapped and depart more from their origin than the $(\mathcal{E B})_{\text {STO }}$ trajectories. This tendency, observed for the few samples in Figure 6, reflects in a full population average of the mean square displacement (Fig. 7). Here we see that the $(\mathcal{E B})_{\text {DNS }}$ average grows somewhat faster than the $(\mathcal{E B})_{\text {STO }}$ average during the first 200 gyro times, from which point on a relative excess of about $30 \%$ is preserved up to $t / t_{C} \sim 10^{4}$. During this time interval, the mean-square displacement grows approximately linearly with time (dashed). Later on, the $(\mathcal{E B})_{\text {STO }}$ curve overtakes the $(\mathcal{E B})_{\mathrm{DNS}}$ curve and grows again faster than linearly with time. The bends observed in Figure 7 indicate changes of acceleration regimes, which are discussed below.

The distance traveled by the test particles ultimately exceeds the side length of the MHD simulation cube, and the particles cross many periodic continuations of this cube. However, the particle orbits themselves are not periodic, because opposite points such as $(x, y$, and 0$)$ and $\left(x, y\right.$, and $\left.l_{z}\right)$ are not magnetically connected (this is a consequence of the fully three-dimensional nature of the MHD simulation) and because the particles may leave their magnetic field lines. Rather, the particle orbits have mixing and ergodic properties. Therefore, the periodicity of the MHD fields is not expected to affect the particle results.

\subsection{Momentum Diffusion}

Next we consider the diffusion in momentum space (momentum $\boldsymbol{p}=\gamma \boldsymbol{v}$ is a better indicator for diffusion than the velocity because it is not limited by the speed of light). The evolution of the mean square momentum is shown in Figure 8 for both $(\mathcal{E B})_{\text {DNS }}$ and $(\mathcal{E B})_{\text {STO }}$ ensembles. The $(\mathcal{E B})_{\text {DNS }}$ curve grows faster at $10<t / t_{C}<10^{2}$, which is the typical particle crossing time of the coherent structures. Later, when many coherent structures have been visited $\left(t / t_{C} \sim 5 \times 10^{3}\right)$, the $(\mathcal{E B})_{\text {DNS }}$ curve is overtaken by $(\mathcal{E B})_{\text {STO }}$. The average terminal $\left(t / t_{C}=1.9 \times 10^{4}\right)$ energy is $\langle\langle\gamma\rangle\rangle=6.4$ for the DNS case and $\langle\langle\gamma\rangle\rangle=8.0$ for the STO case. Thus our protons are accelerated to relativistic energies.

\subsection{Momentum-Displacement Correlation}

The propagation of diffusion from momentum space [where $(\mathcal{E B})_{\text {DNS }}$ and $(\mathcal{E B})_{\text {STO }}$ act] into real-space is governed by the mixed 

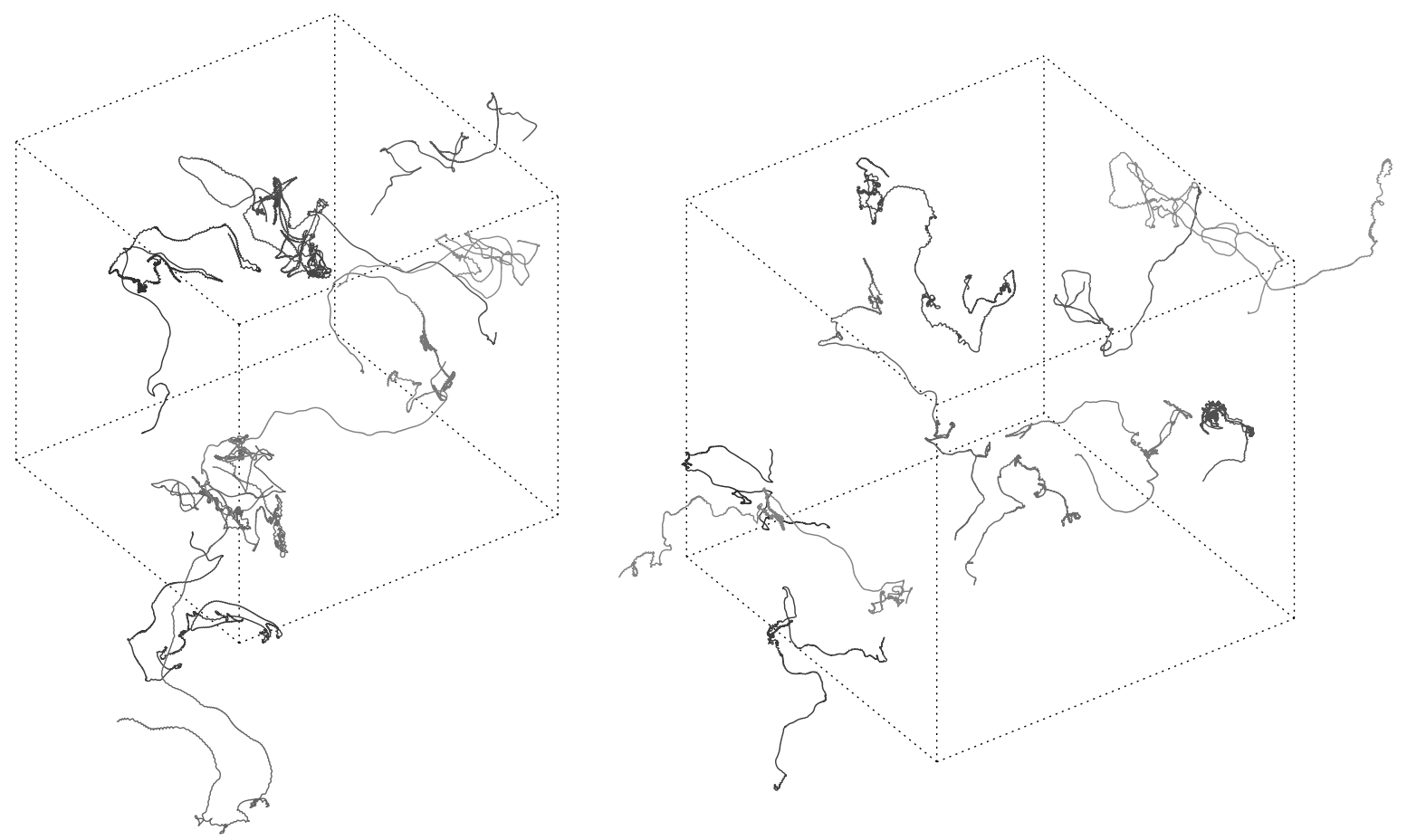

FIG. 6.- Real-space orbits in $(\mathcal{E B})_{\mathrm{DNS}}($ left $)$ and $(\mathcal{E B})_{\mathrm{STO}}($ right $)$ fields for $0 \leq t / t_{C} \leq 1.5 \times 10^{3}$. The gyration is not resolved in this view.

correlation $(d / d t)\left\langle\left\langle|\boldsymbol{x}(t)-\boldsymbol{x}(0)|^{2}\right\rangle\right\rangle=2\langle\langle[\boldsymbol{x}(t)-\boldsymbol{x}(0)] \cdot \boldsymbol{v}(t)\rangle\rangle$. This quantity is always positive due to the statistical preference for particles having traveled away from the origin to have a velocity component in the same direction. Since the coherent structures are able to channel the particles along filaments, they impose a correlation between position and momentum, and we may expect $\langle\langle[\boldsymbol{x}(t)-\boldsymbol{x}(0)] \cdot \boldsymbol{p}(t)\rangle\rangle$ to grow faster in $(\mathcal{E B})_{\mathrm{DNS}}$ than in $(\mathcal{E B})_{\mathrm{STO}}$ during the first structure travel time. This is indeed the case, as shown in Figure 9. The plateau at intermediate times $100 \leq$ $t / t_{C} \lesssim 2000$ indicates the regime of ordinary diffusion.

\subsection{Energy Spectrum}

The evolution of the full energy distribution is shown in Figure 10. The particles start out at $t=0$ with kinetic energy $0.0037 \mathrm{mc}^{2}$ and spread then to higher values. Note that for $t / t_{C} \lesssim 3 \times 10^{3}$, the DNS fields are more efficient accelerators than

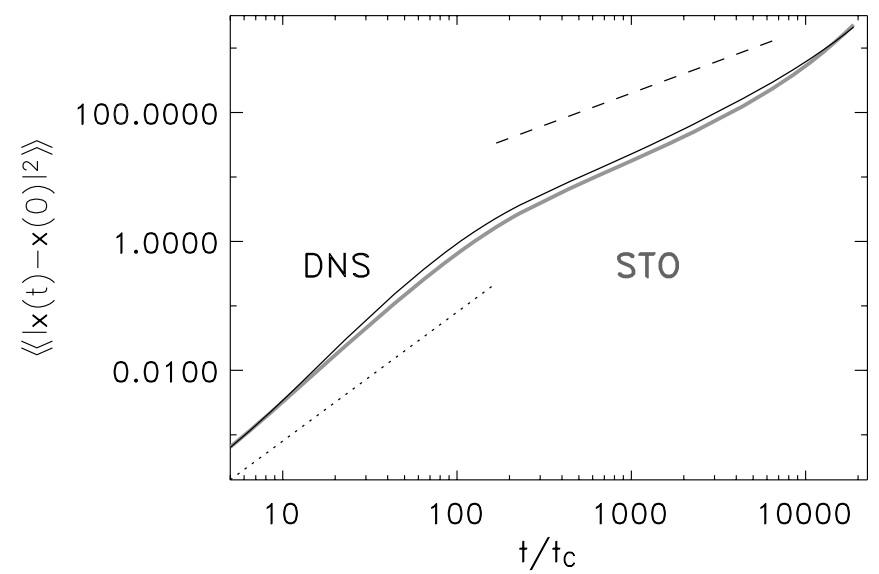

FIG. 7.-Mean square displacement in correlated (black) and decorrelated (gray) fields. The dashed and dotted lines indicate linear and quadratic growth, respectively. the STO fields, while the converse holds true at later times. The spectral shape has slightly positive (negative) curvature for $t / t_{C} \lesssim$ $5 \times 10^{3}\left(t / t_{C} \gtrsim 5 \times 10^{3}\right)$, indicating that the distribution functions decay subexponentially (superexponentially). When fitted by a power law $N(E) \sim E^{-\xi}$ in the subexponential range $400<t / t_{C}<$ 2000 , power-law indices $\xi=4.5, \ldots, 3.7$ are found. Similar exponents have been observed in medium-size solar flares, although for electrons, and smaller energies $E / m_{e} c^{2} \lesssim 0.2$ (Grigis \& Benz 2004).

\section{ACCELERATION MECHANISM}

The ultimate cause of particle acceleration is the electric field, which is now examined in some detail together with the microscopic nature of the particle orbits. From a particle dynamics point of view, the main classification of $\boldsymbol{e}$ is into potential-solenoidal and parallel-perpendicular (to $\boldsymbol{b}$ ) components. From an MHD point of view, one may further distinguish between convective and dissipative fields.

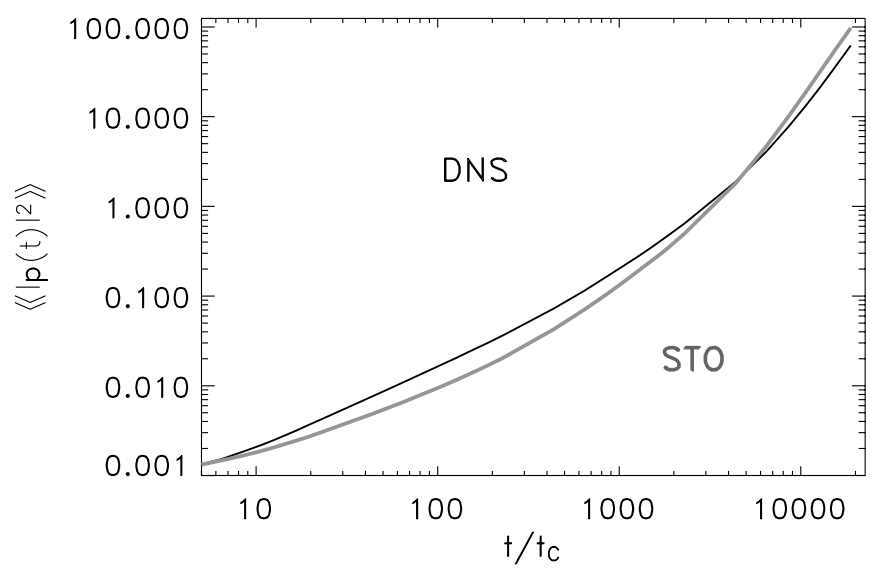

FIG. 8.-Mean square momentum in correlated (black) and decorrelated (gray) fields. 


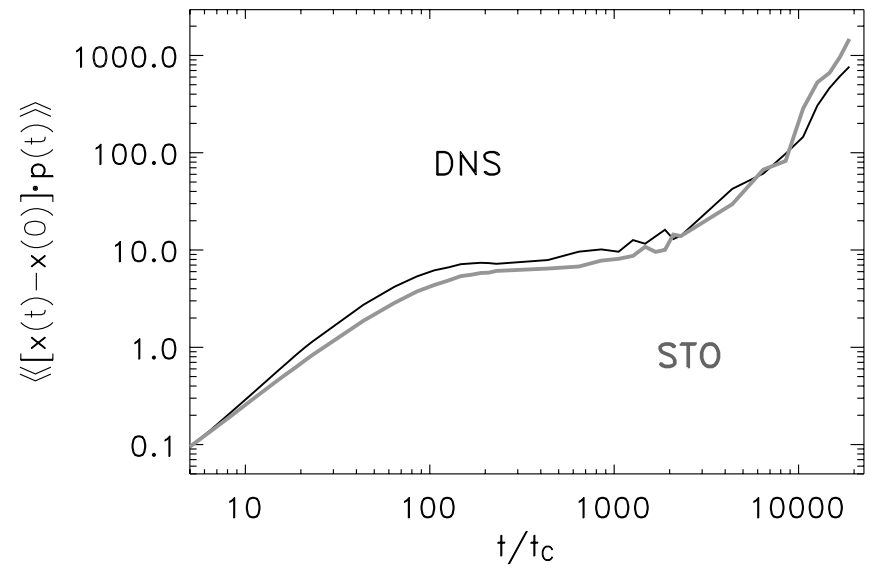

FIG. 9.-Displacement-momentum correlation.

\subsection{Solenoidal and Potential Electric Fields}

By virtue of the "snapshot" condition (eq. [8]), the potential part $-\nabla \phi$ of the electric field does not contribute to acceleration on timescales that are long compared to the crossing time of local potential minima. However, the initially spatially uniform distribution of particles may become nonuniform at later times, resulting in a shift of the average kinetic energy which would manifest in Figures 8 and 10. This shift is bounded by the magnitude of the potential fluctuations $\Delta \gamma=\alpha \Delta \phi / c^{2} \lesssim 0.08$, which is small compared to the energies reached in the tail of the energy distribution (Fig. 10). It is thus the solenoidal electric field $\boldsymbol{e}_{\text {sol }}$ that is responsible for acceleration. From an MHD point of view, $\boldsymbol{e}_{\text {sol }}$ is made up of the dissipative field $\eta \boldsymbol{j}$ plus parts of the convective field $-\boldsymbol{u} \times \boldsymbol{b}$. Numerically, the individual (rms) contributions are $e_{\text {sol,conv }}^{\mathrm{DNS}}=8.0, e_{\mathrm{sol}, \mathrm{Sonv}}^{\mathrm{STO}}=9.35$, and $e_{\mathrm{sol}, \mathrm{Diss}}^{\mathrm{DNS}}=e_{\mathrm{sol}, \text { diss }}^{\mathrm{STO}}=0.35$. Thus, the solenoidal electric field is somewhat weaker in the DNS than in the STO case, in contrast to the enhanced DNS acceleration efficiency at $t / t_{C} \lesssim 5 \times 10^{3}$.

\subsection{Parallel and Perpendicular Electric Fields}

The picture, though, changes if we distinguish between parallel and perpendicular components, where $e_{\mathrm{sol}, \|}^{\mathrm{DNS}}=0.24>e_{\mathrm{sol}, \|}^{\mathrm{STO}}=$

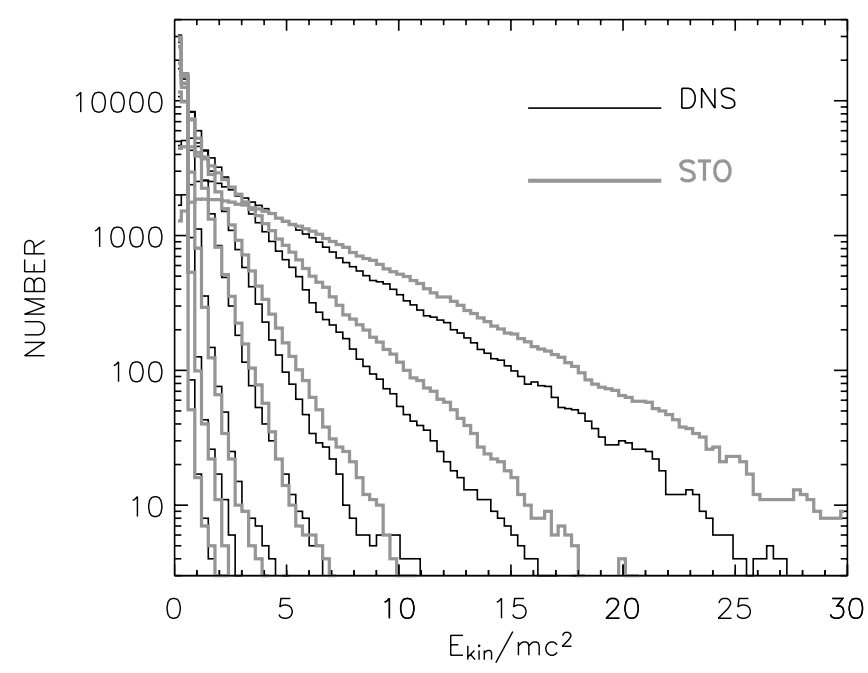

FIG. 10.-Energy distributions at $t / t_{C}=643,1264,2299,4369,6439$, 10,579 , and 16,789 (left to right).

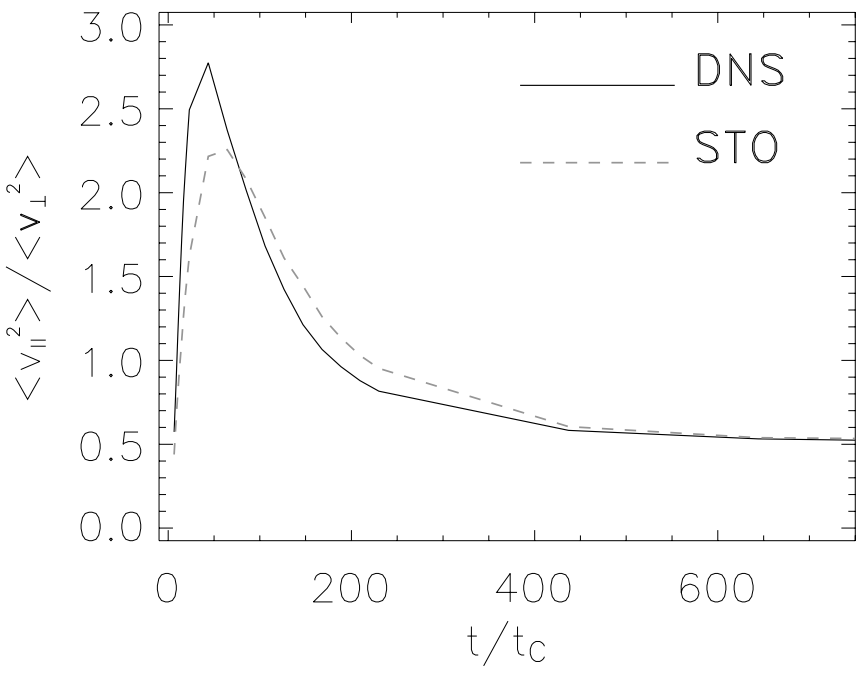

FIG. 11.-Particle anisotropy in the $(\mathcal{E B})_{\mathrm{DNS}}$ and $(\mathcal{E B})_{\mathrm{STO}}$ fields.

0.20 . Initially $(t=0)$, the Larmor radius is smaller than the size of the magnetic inhomogeneities, so that the particles are in a gyrokinetic regime. The ratio of the (rms) forces on the particles is $\mid \boldsymbol{v} \times$ $\boldsymbol{b}|/| \boldsymbol{e}_{\perp}|/| \boldsymbol{e}_{\|} \mid \sim 1.7 / 12 / 0.3$, so that the particles, typically, exhibit a strong $\boldsymbol{e} \times \boldsymbol{b}$ drift. The drift velocity $\boldsymbol{v}_{d}=\boldsymbol{e} \times \boldsymbol{b} /|\boldsymbol{b}|^{2}$ thus approaches $v_{\mathrm{A}}$, and the maximal drift Larmor radius $r_{\mathrm{L}}^{*} \doteq \frac{1}{2} \alpha\left|\boldsymbol{e}_{\perp}\right|\left(t_{C} / 2\right)^{2}$ exceeds its initial (thermal) value $v_{\perp} /(\alpha b)$ by up to an order of magnitude. This is a consequence of the strong turbulence assumption, where $v_{\mathrm{A}}$ is given by the perturbations and not by a (dominant) background magnetic fields. Although the particles gain substantial perpendicular momentum during the first half gyration, this is approximately restored in the second half, and so forth, so that there is little net gain from $\boldsymbol{e}_{\perp}$. The parallel field $\boldsymbol{e}_{\|}$, however, yields direct parallel acceleration. As a consequence, the velocity distribution becomes anisotropic (Fig. 11), with a maximum anisotropy reached at $t / t_{C} \sim 50$. At later times, the velocities isotropize. One reason for this is the (reversible) conversion of parallel into perpendicular momentum at converging magnetic field lines. This mechanism is supported by the observation that $\left\langle\left\langle|\boldsymbol{b}|^{2}\right\rangle\right\rangle$ is slightly $\left(0.04^{2}\right)$ enhanced during the isotropization phase $30 \lesssim$ $t / t_{C} \lesssim 300$, as would be expected when particles penetrate high- $|\boldsymbol{b}|$ domains.

A second cause of the isotropization observed in Figure 11 is the fact that the gyrokinetic approximation breaks down with increasing energy. In order to monitor the transition from gyrokinetic to eventually free orbits we compare the orbital radius of curvature $\rho=1 / K$ to the characteristic scales of the magnetic field, where $K^{2}=\left(|\dot{\boldsymbol{x}}|^{2}|\ddot{\boldsymbol{x}}|^{2}-|\dot{\boldsymbol{x}} \cdot \ddot{\boldsymbol{x}}|^{2}\right) /|\dot{\boldsymbol{x}}|^{3}$. The evolution of $\rho$ is shown in Figure 12 for a sample of 50 particles (dots), with the average $\langle\langle\rho\rangle\rangle$ marked by solid line. Initially, $\langle\langle\rho\rangle\rangle$ is smaller than the Taylor microscale $\lambda_{b}$ and also smaller than the numerical cell $\delta$ (the electromagnetic fields are interpolated to the actual particle position). It then rapidly grows during $0<t / t_{C} \lesssim 50$, where it approaches the cell size and the growth slows down. The quantity $r_{\mathrm{L}}^{*}$ defined in the foregoing paragraph is found equal to the maximum of $\rho$ during the first gyration within $\pm 10 \%$. Based on Figure 12 and the criterion $\langle\langle\rho\rangle\rangle>\delta$ one may infer that, on average, the gyrokinetic regime is left after a few hundred gyro times. At this point the character of the orbit and the acceleration mechanism change.

A picture thus arises in which acceleration is initiated by the parallel component of the solenoidal electric field ("injection") 


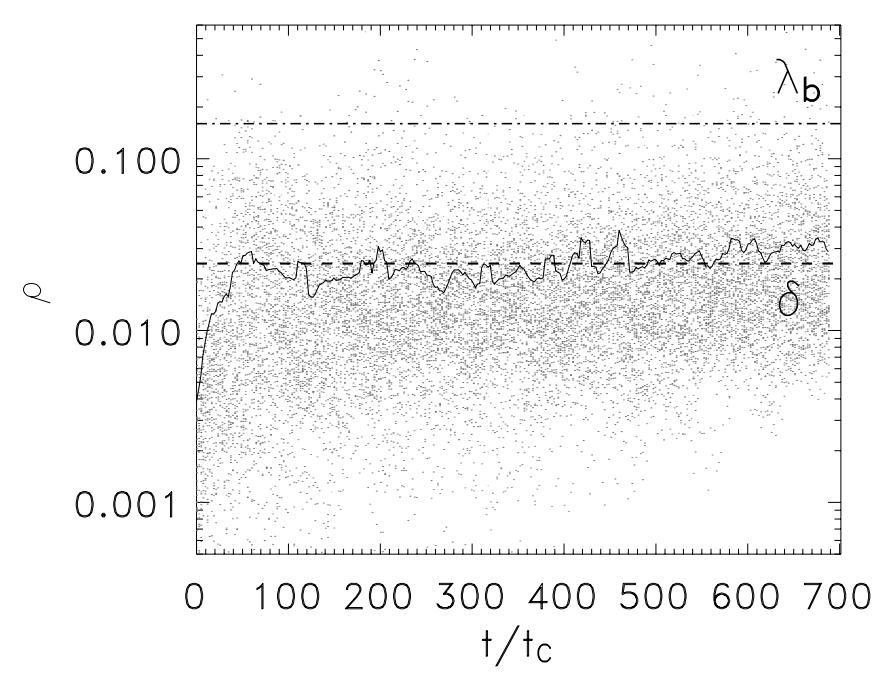

FIG. 12.- Orbital radius of curvature vs. time: sample orbits (dots); average (solid line). For comparison, the numerical cell $\delta$ and the Taylor microscale $\lambda_{b}$ are also indicated.

and is then taken over by the full nonpotential electric field. While the parallel component is somewhat larger in $(\mathcal{E B})_{\mathrm{DNS}}$, the total solenoidal field is larger in $(\mathcal{E B})_{\mathrm{STO}}$. Thus, the DNS fields are initially stronger accelerators, but the STO fields become more efficient at a later (nonadiabatic) stage. In both cases, the parallel electric field is entirely dissipative. The initial (parallel) acceleration phase corresponds to the first phase of Figure $7\left(t / t_{C} \lesssim 200\right)$.

The fact that the parallel field is weakened by the phase randomization, while the perpendicular electric field is strengthened, is easily understood from Figure 4. The nonlinear MHD alignment of $\boldsymbol{b}$ with $\boldsymbol{j}$ favors small $\left|j_{\|}\right|$; this preference is lost in the phase randomization so that $\left|j_{\|}\right|$increases. Similarly, the MHD alignment of $\boldsymbol{u}$ and $\boldsymbol{b}$ is lost in the phase randomization, and $|\boldsymbol{u} \times \boldsymbol{b}|$ increases. Since the convective field numerically dominates the dissipative one, the solenoidal electric field as a whole increases when the phase randomization is applied to $\boldsymbol{u}$ and $\boldsymbol{b}$.

\subsection{Direct Phase Randomization of $\boldsymbol{e}$}

The crucial role of parallel electric fields can also be demonstrated by an alternative simulation setup, in which $\boldsymbol{e}$ is directly phase randomized rather than computed from the phase-randomized $\boldsymbol{u}$ and $\boldsymbol{b}$. The ideal MHD condition $\boldsymbol{e} \cdot \boldsymbol{b}=0$ is then broken even in the absence of resistivity, while the power spectral densities of $\boldsymbol{b}$ and $\boldsymbol{e}$ remain unchanged. We may thus ask what happens to the particles in such a directly phase-randomized electric field. This procedure has a certain interest on its own because of its implication on random-phase Fokker-Planck descriptions of stochastic acceleration.

It turns out that the direct phase randomization of $\boldsymbol{e}$ leads to a dramatic enhancement of acceleration. Figure 13 shows the evolution of $\left\langle\left\langle|\boldsymbol{p}(t)-\boldsymbol{p}(0)|^{2}\right\rangle\right\rangle$ for the DNS and directly phaserandomized cases. The curve corresponding to direct phase randomization of $\boldsymbol{e}$ is labeled "STO (E)." As can be seen, the DNS and STO (E) curves separate after about half a gyro period, and the direct phase randomization of the electric field yields energies that are 2 orders of magnitude above the $(\mathcal{E B})_{\text {STO }}$ results. This dramatic enhancement of acceleration is entirely due to (unphysical) parallel electric fields. The local maximum of the DNS curve at $t_{C} / 2$ marks the first half gyro phase, where the velocities are, on average, reversed. At this point, the particles in the directly phase-randomized electric field have already gained super-

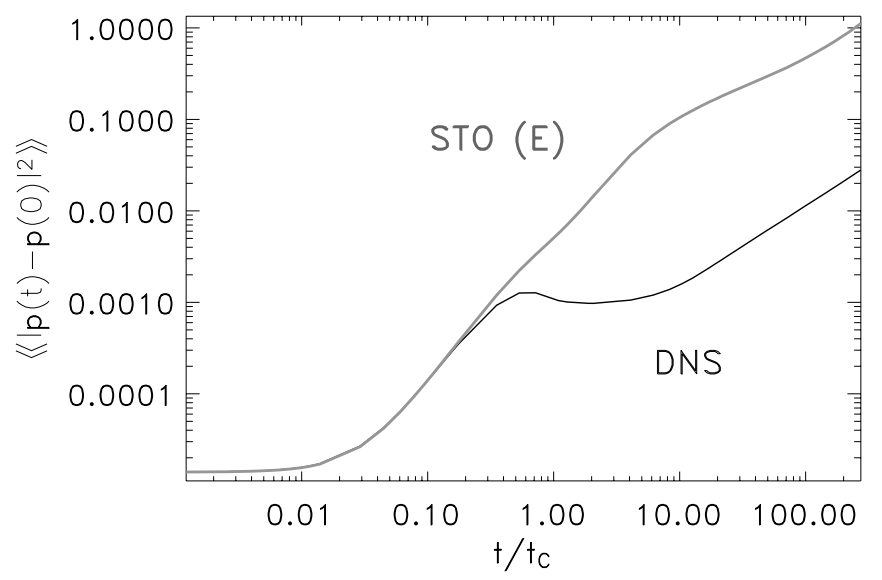

FIG. 13.-Similar to Fig. 8, but with $\boldsymbol{e}$ directly phase randomized (instead of being computing from the phase randomized $\boldsymbol{b}$ and $\boldsymbol{u}$ ). The time axis spans a smaller range than in Fig. 8.

Alfvénic velocities, so that the effect of gyration is no longer visible.

\section{SUMMARY AND DISCUSSION}

We have performed a numerical study of test particle acceleration in strong MHD turbulence $(\boldsymbol{b}, \boldsymbol{u})$ and its phase-randomized version, with the electric field given by $\boldsymbol{e}=-\boldsymbol{u} \times \boldsymbol{b}+\eta \boldsymbol{j}$. It is found that the dynamical alignment of $\boldsymbol{b}$ with $\boldsymbol{j}$ and $\boldsymbol{u}$ in true MHD affects the division of the electric field into parallel and perpendicular components, in the sense that parallel (resistive) components are favoured and perpendicular (convective) components are disfavored. As a consequence, the true MHD turbulence provides more efficient direct acceleration of super-Dreicer particles than its phase-randomized version. If the particles reach sufficiently high energies to leave the gyrokinetic regime, so that they can become accelerated by the solenoidal part of the $\boldsymbol{u} \times \boldsymbol{b}$ force, the dynamical alignment of $\boldsymbol{u}$ with $\boldsymbol{b}$ yields weaker acceleration than predicted from the phase-randomized fields. For collisionless protons in MHD turbulence with magnetic Reynolds number $\sim 300$, this is the case for protons after a few 1000 gyro times. Numerically, the acceleration efficiencies of the true MHD and phase-randomized fields differ by factors of order 2 . It is expected that the discrepancy would be larger for super-Dreicer electrons, since these remain longer in the adiabatic regime in which the enhancing effect of $(\boldsymbol{b}, \boldsymbol{j})$ alignment is not yet competed with the diminishing effect of $(\boldsymbol{b}, \boldsymbol{u})$ alignment.

With regard to solar flares envisaged as a astrophysical application, a few words of caution are appropriate. The present simulation involves only dimensionless quantities such as $r_{\mathrm{L}} / \lambda_{b}$ or $r_{\mathrm{L}} / \delta$. In order to fix the absolute physical scaling, and since we were interested in the possibility of nonadiabatic acceleration, we have chosen protons as test particle. Using typical values of the coronal magnetic field strength and taking the resolution of the simulation into account, the protons become nonadiabatic $\left(r_{\mathrm{L}}>\delta\right)$ at $v \gtrsim 0.6 c$ and reach energies as high as $10 \mathrm{GeV}$ (10 proton rest masses). Such high energies are commonly not observed; the highest energies from in situ observations in the interplanetary space are about $100 \mathrm{MeV} /$ nucleon (Reames et al. 1992), and proton energies inferred from pion decay photons reach up to a few $100 \mathrm{MeV} /$ nucleon and occasionally up to a few giga-electron volts (Kanbach et al. 1993). (Only in the very largest flares such as of 2003 October 28, ground-based detections of relativistic protons and neutrons show energies up to $10 \mathrm{GeV}$ [Bieber et al. 2004].) Also, the simulated acceleration is very 
rapid; assuming $\left\langle|\boldsymbol{b}|^{2}\right\rangle \sim(100 G)^{2}$, the simulation duration is only $0.1 \mathrm{~s}$ in real time. There are two main reasons for the violent acceleration in the present simulation: the neglect of collisional and radiative losses and the freedom in the absolute scaling of the simulation system. The latter is chosen such that one numerical cell is about 200 initial $\left(|\boldsymbol{v}|=v_{0}\right)$ Larmor radii; this corresponds to $\delta \sim$ $100 \mathrm{~m}$ in real space. The resulting steep gradients - which are not detectable by present-day observations - together with a larger than coronal resistivity $\left(\operatorname{Re}_{b} \sim 300\right)$, yield highly efficient accelerators, which allow us to rigorously follow the particles to relativistic energies before becoming swamped by accumulated numerical errors (and running out of computing time). The simulation system is thus affected by computational considerations and presumably overestimates the absolute coronal acceleration rate. However, the relative influence of dynamical MHD alignment on particle acceleration should be correctly reproduced.

Another caveat stems from the test particle nature of the present simulation, which does not allow to address the question of absolute numbers of accelerated particles. The test particles are considered as resulting from a runaway process that is out of the scope of this simulation, so that we cannot directly relate their number to the number of thermal background particles. However, it is clear that the test particles must not gain more energy than is available in the MHD fields. This sets an upper limit on their number and also on the duration of the simulation. (As the simulation does not include loss processes, the test particles can gain arbitrary large energy.) In our simulations, the test protons pick up Alfvénic velocities within one gyration. This brings them to kinetic energies of the ambient (low-beta) coronal plasma, which is assumed to be in equipartition, $\left\langle|\boldsymbol{u}|^{2}\right\rangle=\left\langle|\boldsymbol{b}|^{2}\right\rangle$. In the course of the simulation, the kinetic energy of the test particles increases by a factor $10^{4}$. This should not exceed a small fraction (say, $\epsilon=10^{-2}$ ) of the magnetic field energy, so as not to perturb the MHD configuration. Therefore, the test particles represent a fraction $f=10^{-6}$ of all particles only. This fraction is small, but not unreasonably small for runaway protons, which must be substantially faster than the electrons in order for runaway to occur (Dreicer 1960). It is also in agreement with the observation that most solar flares do not show a signature of high-energy protons (but only of high-energy electrons). However, if the test particles are to represent more than $10^{-6}$ of all particles, then the acceleration should be stopped at earlier times. Since it is found that the test particle energy increases roughly linearly with time, the maximum simulation duration is $t_{\max } / t_{C} \sim \epsilon / f$. In fact, it might happen that the MHD description brakes down after a few 100 gyro times, so that the nonadiabatic regime, in which the $(\mathcal{E B})_{\mathrm{STO}}$ fields become more efficient accelerators, is never reached.

Returning to the more technical aspects and motivated by the findings of $\S 5.3$, we would like to stress that violation of the ideal MHD condition $\boldsymbol{b} \cdot \boldsymbol{e}=0$ by random-phase approximations unavoidably leads to artificial particle acceleration. If, in theory or numerics, a turbulence proxy is generated by superposing Alfvén modes $\left(\delta \boldsymbol{b}_{\boldsymbol{k}}, \delta \boldsymbol{e}_{\boldsymbol{k}}\right) e^{i \boldsymbol{k} \cdot \boldsymbol{x}-i\left(\boldsymbol{k} \cdot \boldsymbol{b}_{0}\right) t}$ with $\delta \boldsymbol{e}_{k}=\boldsymbol{b}_{0} \times \delta \boldsymbol{b}_{\boldsymbol{k}}$, then a parallel electric field occurs which is of second order in $\delta b$. For example, if the $\delta \boldsymbol{b}_{\boldsymbol{k}}$ are random isotropic in the plane perpendicular to $\boldsymbol{b}_{0}$, with orientation uncorrelated to $\boldsymbol{k}$, then $\left\langle(\boldsymbol{e} \cdot \boldsymbol{b})^{2}\right\rangle=$ $\frac{1}{4} b_{0}^{2}\left\langle(\delta b)^{2}\right\rangle^{2}$; for $\delta b \ll b_{0}$, the parallel electric field has thus standard deviation $\frac{1}{2}\left\langle(\delta b)^{2}\right\rangle$. Although this is small compared to the perpendicular electric field $\left(e_{\|} / e_{\perp} \sim \delta b / b_{0}\right)$, it may give rise to secular growth of the parallel particle momentum. Therefore, stochastic acceleration in turbulence proxies constructed from superposed Alfvén waves might in fact overestimate the acceleration in true MHD turbulence.

Finally, we point out that although the coherent structures do not manifest in the two-point functions (or power spectral densities) of $\boldsymbol{b}$ and $\boldsymbol{e}$ alone, they contribute to their mixed correlations. This fact can, in principle, be taken into account in Langevin or Fokker-Planck formulations of stochastic acceleration (e.g., Brissaud \& Frisch 1974; Van Kampen 1981; Risken 1989; Urch (1977); Urch (1991); Gardiner 1997; Schlickeiser \& Miller (1998); Schlickeiser (2002); Falkovich et al. 2001), which deal with the transfer of spatial correlators of the electromagnetic fields into the particle diffusion coefficients. Given the correlations $\left\langle b_{i}(0)-b_{j}(\boldsymbol{r})\right\rangle,\left\langle b_{i}(0)-e_{j}(\boldsymbol{r})\right\rangle$, and $\left\langle e_{i}(0)-e_{j}(\boldsymbol{r})\right\rangle$, one may compute, for example, the momentum diffusion coefficient $D_{p p}=$ $\int_{0}^{\infty}\langle\langle\delta \boldsymbol{f}(0) \cdot \delta \boldsymbol{f}(\tau)\rangle\rangle d \tau$ along the drifting gyrocenter $\boldsymbol{x}_{0}(\tau)=$ $\boldsymbol{x}(0)+v_{\|}(0) \hat{b} \tau+\boldsymbol{v}_{d} \tau(\S 5.2)$, where $\delta \boldsymbol{f}=\boldsymbol{e}(\boldsymbol{x})+\boldsymbol{v} \times \boldsymbol{b}(\boldsymbol{x})$ for strong turbulence. The quantity $D_{p p}$ may then be evaluated numerically, as follows. First we choose a large $\left(10^{7}\right)$ number of random initial data $[\boldsymbol{x}(0), \boldsymbol{v}(0)]$ with uniform position $\boldsymbol{x}(0)$ and isotropic velocity of fixed modulus $|\boldsymbol{v}(0)|=v_{0}=0.2 v_{\mathrm{A}}$, like for the simulated test particles. Then, for each $[x(0), v(0)]$ the instantaneous (straight) gyrocenter orbit $\boldsymbol{x}_{0}(\tau)$ is computed, and the electromagnetic force $\delta \boldsymbol{f}(\tau)$ is probed along this orbit. The average of $\langle\langle\delta \boldsymbol{f}(0) \cdot \delta \boldsymbol{f}(\tau)\rangle\rangle$ over all particles (initial data) is performed, and the integral in the definition of $D_{p p}$ is approximated by a sum of sufficiently resolved increments. Proceeding this way, we obtain $D_{p p}=0.03$ for $(\mathcal{E B})_{\text {DNS }}$ and $D_{p p}=0.018$ for $(\mathcal{E B})_{\text {STO }}$. The ratio of these values reflects the excess of $\left\langle\left\langle p^{2}\right\rangle\right\rangle_{\mathrm{DNS}}$ over $\left\langle\left\langle p^{2}\right\rangle\right\rangle_{\text {STO }}$ observed in Figure 8 for $t / t_{C} \lesssim 1000$. A detailed study of the modification of $D_{p p}$ by the cross-correlation $\left\langle b_{i}(0)-e_{j}(\boldsymbol{r})\right\rangle$ has, to the authors knowledge, not yet been carried out.

This work was supported in part by the Research Training Network (RTN), Theory, Observation and Simulation of Turbulence in Space Plasmas, funded by the European Commission (contract HPRN-eT-2001-00310).

\section{REFERENCES}

Achterberg, A. 1981, A\&A, 97, 259

Adler, R. J. 1981, The Geometry of Random Fields (New York: John Wiley \& Sons) Arzner, K., \& Vlahos L. 2004, ApJ, 605, L69

Arzner, K., Vlahos, L., Knaepen, B., \& Denewet, N. 2005, Springer Lecture Notes in Computer Science 3723 (Berlin: Springer), 538

Batchelor, G. K. 1982, The Theory of Homogeneous Turbulence (Cambridge: Cambridge Univ. Press)

Benz, A. 2003, in Energy Conversion and Particle Acceleration in the Solar Corona, ed. L. Klein (Berlin: Springer)

Bieber, J. W., Clem, J., Evenson, P., Pyle, R., Ruffolo, D., \& Saiz, A. 2004, Geophys. Res. Lett., 32, L03S02

Biskamp, D. 1993, Nonlinear Magnetohydrodynamics (Cambridge: Cambridge Univ. Press)

Biskamp, D., \& Müller, W. 1999, Phys. Rev. Lett., 83, 2195
Brissaud, A., \& Frisch, U. 1974, J. Math. Phys., 15, 624

Büchner, J., \& Zelenyi, L. M. 1989, J. Geoph. Res., 94, 11.821

Bykov, A. M., \& Toptygin, I. N. 1993, Phys. Uspekhi, 36 (11), 1020

Cho, J., Lazarian. A., \& Vishniac, E. 2002, ApJ, 564, 291

Comte-Bellot G., \& Corrsin S. 1971, J. Fluid Mech., 48, 273

Dar, G., \& Verma, K. 1998, Phys. Plasmas, 5, 2528

Debliquy, O., Verma, M., \& Carati, D. 2005, Phys. Plasmas, 12, 042309

Dmitruk, P., Mattheus, W. H., Seenu, N., \& Brown, M. R. 2003, ApJ, 597, L81

Dreicer, H. 1959, Phys. Rev., 117, 242 1960, Phys. Rev., 117, 329

Falkovich, G., Gawedzki, K., \& Vergassola, M. 2001, Rev. Mod. Phys., 73 (4), 913

Frisch, U. 1995, Turbulence: The Legacy of A. N. Kolmogoroff (Cambridge: Cambridge Univ. Press) 
Gardiner, C. 1997, Handbook of Stochastic Methods for Physics, Chemistry and the Natural Sciences (2nd ed.; New York: Springer)

Gotoh, T., Fukayama, D., \& Nakano, T. 2002, Phys. Fluids, 14, 1065

Grigis, P., \& Benz, A. 2004, A\&A, 426, 1093

Helander, P., \& Sigmar, D. J. 2002, Collisional Transport in Magnetized Plasmas (Cambridge: Cambridge Univ. Press)

Kanbach, G., et al. 1993, A\&AS, 97, 349

Karimabadi, H., Omidi, N., \& Menyuk, C. R. 1990, Phys. Fluids B, 2, 606

Kolmogoroff, A. N. 1941a, Dokl. Akad. Nauk SSSR, 30, 301

1941b, Dokl. Akad. Nauk SSSR, 32, 16

Littlejohn, R. G. 1982, J. Math. Phys. 23 (5), 742

Mace, R. L., Matthaeus, W. H., \& Bieber, J. 2000, ApJ, 538, 192

Matthaeus, W. H., Ambrosiano, J. J., \& Goldstein, M. L. 1984, Phys. Rev. Lett. 53,1449

McComb, W. D. 1990, The Physics of Fluid Turbulence (Oxford: Oxford Univ. Press)
Miller, J. A., et al. 1997, J. Geoph. Res., 102 (14), 631

Paesold, G., Kallenbach, R., \& Benz, A. O. 2003, ApJ, 582, 495

Pouquet, A., Meneguzzi, M., \& Frisch U. 1986, Phys. Rev. A, 33, 4266

Reames, D. V., Richardson, I. G., \& Wenzel, K. P. 1992, ApJ, 387, 715

Risken, H. 1989, The Fokker-Planck Equation (2nd ed.; Berlin: Springer)

Schlickeiser, R. 2002, Cosmic Ray Astrophysics (Berlin: Springer)

Schlickeiser, R., \& Miller. J. A. 1998, ApJ, 492, 352

Sorriso-Valvo, L., Carbone, V., Veltri, P., Politano, H., \& Pouquet A. 2000, Europhys. Lett., 51, 520

Urch, I. H. 1977, Ap\&SS, 48, 231

1991, Ap\&SS, 178, 185

Van Kampen, N. G. 1981, Stochastic Processes in Physics and Chemistry (Amsterdam: North Holland)

Verma, M. 2004, Phys. Rep., 401, 229 